$\begin{array}{ll} & \text { Etnográfica } \\ \text { etnográfica } & \text { Revista do Centro em Rede de Investigação em }\end{array}$

Antropologia

vol. 24 (3) | 2020

Vol. 24 (3)

\title{
La mémoire des migrations comme support de promotion municipale : la fête de l'émigrant de Murtosa
}

The memory of migrations as a medium for municipal promotion: the emigrant's festivity of Murtosa

\section{Guillaume Etienne}

\section{(2) OpenEdition}

\section{Journals}

Édition électronique

URL : https://journals.openedition.org/etnografica/7808

DOI : $10.4000 /$ etnografica. 7808

ISSN : 2182-2891

\section{Éditeur}

Centro em Rede de Investigação em Antropologia

Édition imprimée

Date de publication : 1 octobre 2020

Pagination : 629-652

ISSN : 0873-6561

Référence électronique

Guillaume Etienne, "La mémoire des migrations comme support de promotion municipale : la fête de l'émigrant de Murtosa », Etnográfica [En ligne], vol. 24 (3) | 2020, mis en ligne le 31 octobre 2020, consulté le 20 janvier 2022. URL : http://journals.openedition.org/etnografica/7808 ; DOI : https:// doi.org/10.4000/etnografica.7808

\section{(c) (7) \&}

Etnográfica is licensed under a Creative Commons Attribution-NonCommercial 4.0 International License. 


\section{La mémoire des migrations comme support de promotion municipale : la fête de l'émigrant de Murtosa}

\section{Guillaume Etienne}

Comment les migrations et les relations transnationales qui en découlent sontelles réinterrogées par les habitants et leurs élus au prisme de sphères fort variées de la vie sociale locale ? Cet article interroge la façon dont des liens transnationaux dépassent les territoires, les villes et les pays concernés pour finalement servir de support à d'autres types de relations, notamment touristiques. Une recherche menée entre la France et le Portugal permet d'éclairer trois aspects de ces liens, articulés autour de la valorisation de l'émigration. Le cas de Murtosa, ville portugaise marquée par une " culture de la migration ", illustre un tel processus d'élargissement des liens. Mon propos présente tout d'abord l'établissement d'un consensus sur le respect des émigrants et son corollaire, moins légitime, des voix remettant en cause ce consensus. Deuxièmement, j'aborde les modalités de création de nouveaux liens, notamment par l'entremise d'une fête de l'émigrant, au-delà des villes concernées par les parcours migratoires classiques. Enfin, l'intégration d'une politique de valorisation de l'émigrant questionne la saisie des migrations et des relations transnationales comme ressource dans des perspectives touristiques et de développement local.

MOTS-CLEFS : migration, tourisme, fête, transnationalisme, Murtosa.

The memory of migrations as a medium for municipal promotion: the emigrant's festivity of Murtosa - How are migration and the resulting transnational relationships re-interpreted by residents and their elected representatives in the prism of diverse spheres of local social life? This article examines how transnational links go beyond the territories, cities and countries concerned and ultimately serve as a basis for other types of relationships, particularly tourism. The case of Murtosa, a city marked by a "culture of migration", illustrates such a process of widening ties. My remarks begin by presenting the establishment of a consensus on respect for emigrants and its corollary, less legitimate, of voices questioning this consensus. Second, I discuss how new links are created, including by means of an "emigrant's festivity", extending beyond the cities involved in traditional migration routes. Finally, the integration of a policy to enhance the value of the emigrant raises the question of how to take on migration and transnational relations as resources in tourism and local development perspectives.

KEYWORDS: migration, tourism, celebration, transnationalism, Murtosa.

ETIENNE, Guillaume (guillaume.etienne@univ-tours.fr) - Département de sociologie, Université de Tours, France. 


\section{INTRODUCTION}

Les relations transnationales entre le Portugal et les pays d'arrivée des émigrants portugais, notamment la France, sont désormais bien documentées. Si les champs étudiés (tels que le patrimoine, le sport, le religieux...) sont bien souvent distingués par nécessité de construction de l'objet de recherche, les études ne manquent pas de souligner leur compénétration.

Les fêtes sont parmi les objets emblématiques de cette compénétration des sphères sociales. Les célébrations et les commémorations, les hommages rendus à telle personnalité, les fêtes locales sont porteuses d'une grande variété de significations et rassemblent de multiples acteurs aux attentes tout aussi multiples. Que l'on pense aux commémorations de la Révolution des CEillets ou à la fête nationale du 10 juin, Dia de Portugal, de Camões e das Comunidades (Feldman-Bianco et Huse 1995), pour ne citer que ces exemples caractéristiques, suffit à montrer la portée politique, religieuse, touristique, économique ou encore artistique que peuvent revêtir de telles célébrations, au Portugal ou ailleurs. A côté de ces grandes expressions patrimoniales, ${ }^{1}$ des manifestations ponctuelles, plus modestes, comme les fêtes locales de l'émigrant, valorisent publiquement les migrations. Mais que produisent-elles au-delà d'une mise en lumière passagère de l'émigration?

Dans le cadre de cet article, je souhaite montrer l'articulation de trois dynamiques, que sont la valorisation de l'émigration, la promotion de la ville dans une perspective de développement, notamment touristique, et le maintien ou la création de liens entre diverses localités du Portugal et du monde. Une expérience de recherche menée entre le centre de la France et le centre du Portugal permet en effet d'illustrer la façon dont la ville de Murtosa, par l'entremise de la fête de l'émigrant, déploie et maintient des liens dans un espace transnational en intégrant dans un même schéma ces trois sphères.

Cet article est organisé en trois parties. Dans la première, je présente la ville de Murtosa en précisant le contexte socio-historique des migrations et la place de ces mémoires migratoires au sein de la vie locale. J'y fais également une brève présentation de la ville française de La Riche et de l'étude sur sa population portugaise m'ayant amené à interroger la construction de liens entre les deux villes. Je m'attache dans la deuxième partie à décrire les modalités de valorisation de la figure du migrant, en m'attardant particulièrement sur la fête de l'émigrant à Murtosa. Ces éléments me conduisent enfin à questionner, dans la troisième partie, la façon dont se créent de nouveaux liens entre deux municipalités et leurs effets, notamment en termes de développement économique ou touristique. 


\section{DU CENTRE DU PORTUGAL AU CENTRE DE LA FRANCE}

\section{La culture de la migration à Murtosa}

L'émigration a particulièrement marqué le concelho de Murtosa, ville d'environ 10.000 habitants actuellement, dans la région centre du Portugal, dans le district d'Aveiro. Sujets d'émigration interne (vers Lisbonne ou Porto), mais surtout externe, les murtoseiros se sont particulièrement dirigés vers les Etats-Unis, et notamment Newark, New Jersey, jumelée avec Murtosa depuis 2001. Cette histoire migratoire est, depuis les années 1980, prise en main par la municipalité qui souhaite rendre hommage à ces émigrants, “ exemples de ténacité et de sacrifice, qui savaient réussir dans les pays d'arrivée, avec toujours un œil sur leur pays d'origine, Murtosa, où année après année, ils sont revenus, souvent en été, pour atténuer leur nostalgie ". ${ }^{2}$

De nombreux indices soulignent aujourd'hui l'histoire migratoire de la ville et la culture de la migration locale (Brettell 2003, 2015 [2000]), comme les inscriptions physiques dans la ville : l'avenida do Emigrante, da cidade de Newark (inaugurée en 2011), le monument aux émigrants (inauguré en 1981) ou les commerces dont le nom évoque l'émigration. ${ }^{3}$ De même, les maisons d'émigrants et les drapeaux en azulejos rappellent les trajectoires des propriétaires, partis au Venezuela ou en France, ou encore les statues de la liberté trônant dans les jardins de ceux partis aux Etats-Unis. Des initiatives plus ponctuelles de la mairie, des associations ou du clergé sont d'autres indices, révélant l'importance du phénomène dans la vie locale : fêtes de l'émigrant, courses cyclistes des émigrants, messes des émigrants, portraits d'émigrants filmés. Toutes ces références à la migration, dans le bâti, inscrites dans les généalogies familiales et dans les événements associatifs, culturels, sportifs, fondent depuis plusieurs décennies une culture de la migration, désormais assumée et valorisée par la municipalité.

Le concept de "culture de la migration " caractérise l'ancrage de la migration dans les valeurs locales, ce qui, à Murtosa, s'avère particulièrement pertinent. ${ }^{4}$ Caroline Brettell (2003, 2015 [2000]) emprunte ce concept aux auteurs travaillant sur les migrations mexicaines (Kandel et Massey 2002 ; Cohen

2 Dans l'original portugais : "Exemplos de tenacidade e sacrifício, souberam singrar nas Terras de acolhimento, sempre com os olhos postos na sua Terra de origem, a Murtosa, onde, ano após ano, foram regressando, normalmente no Verão, para mitigar as saudades" (<http://www.cm-murtosa.pt/Templates /GenericDetails.aspx?id_object $=5360>$ (dernière consultation en décembre de 2017).

3 Le monument aux émigrants a été sculpté par José João Brito. Plusieurs études s'attachent à l'analyse des monuments aux émigrants construits dès les années 1980 dans de nombreuses villes portugaises, notamment Tomé, Carreira et Carreira (2000) et Tomé (2009), selon lesquels ces monuments affirmant l'héroïsme des migrants naitraient de l'éloignement de l'idée de retour au pays. Pourtant nombre d'anciens migrants retournent dans leur village d'origine lors de leur retraite.

4 "The 'culture of migration' perspective steers us to a consideration of the embeddedness of migration in local values" (Brettell 2015 [2000] : 156). 
2004) qui en démontrent l'opérativité en tant que la migration des uns modifie les représentations culturelles de ceux qui restent, notamment au regard de la possibilité de migrer comme option. Une histoire migratoire locale, dont la gestion relève autant du national que de l'international (Brettell 2008 : 54), est tellement ancrée dans la vie quotidienne qu'elle s'intègre alors aux schémas culturels existants. Si cette culture de la migration en devient parfois normative, la décision de migrer n'a toutefois rien d'automatique, et les individus envisagent cette possibilité au même titre qu'une autre pour prétendre à une vie meilleure. Nous pouvons considérer, au vu du passé migratoire et de l'ampleur du phénomène à Murtosa, la portée analytique de ce concept dans notre étude. En effet, la tradition migratoire de Murtosa, dont nous allons préciser les détails, s'est peu à peu forgée tout au long du XXe siècle. Les murtoseiros voient alors partir des membres de leur famille, des voisins, des amis, et entendent les récits de réussite sociale et économique qui prédisposent les jeunes gens à recourir, eux aussi, à cette possibilité.

Le concelho de Murtosa, autonome depuis 1926, ${ }^{5}$ comporte quatre freguesias situées de part et d'autre de la Ria de Aveiro : Monte, Murtosa et Bunheiro à l'est de la lagune et Torreira à l'ouest, côté atlantique. ${ }^{6}$ Les principaux secteurs professionnels sont l'agriculture, essentiellement la culture du maïs, et les activités maritimes : pêche, récolte des moliços (algues utilisées pour fertiliser les terres), saliculture, puis commerce maritime et construction navale. En 1960, 61\% de la population active travaillaient dans le secteur primaire, pêche ou agriculture ; seulement 16\% le font aujourd'hui, majoritairement des hommes. ${ }^{7}$ Cependant, la municipalité estime que " les données du recensement ne reflètent pas l'importance du secteur primaire à Murtosa. [...] Malgré cela, il est vrai que le secteur primaire concerne encore un nombre important d'actifs ". 8

L'émigration se constate dès le début du XXe siècle avec un accroissement notable à partir des années 1950, comme l'a souligné Jorge Arroteia. Si, dans la période 1955-59, la première destination est le Venezuela, c'est vers les EtatsUnis que se dirigent ensuite les murtoseiros à partir de 1960. Ainsi, entre 1955 et 1974, 48,8\% des émigrants se sont dirigés vers les Etats-Unis, 20\% vers le Venezuela et 9,1\% vers le Brésil (Arroteia 1984a : 109). A partir de 1965, de

5 Murtosa et Bunheiro dépendaient alors du concelho d'Estarreja.

6 En 2001, Bunheiro comptait 2707 habitants ; Monte, 1116 ; Murtosa, 3140 ; et Torreira, 2495.

7 Voir < https://www.pordata.pt/Municipios/Popula\%c3\%a7\%c3\%a3o+empregada+segundo+os+ Censos + total $+\mathrm{e}+$ por + sector + de + actividade + econ $\%$ c3\%b3mica- $145>$ (dernière consultation en décembre de 2019).

8 "Embora os dados do Censos 2001 não traduzam a importância que o sector primário tem no concelho da Murtosa, uma vez que o mesmo aparece em último lugar, como o que emprega menos população activa, a verdade é que apesar disso, ainda é significativo o número de activos no sector primário" (RSM $2006: 61$ ). 


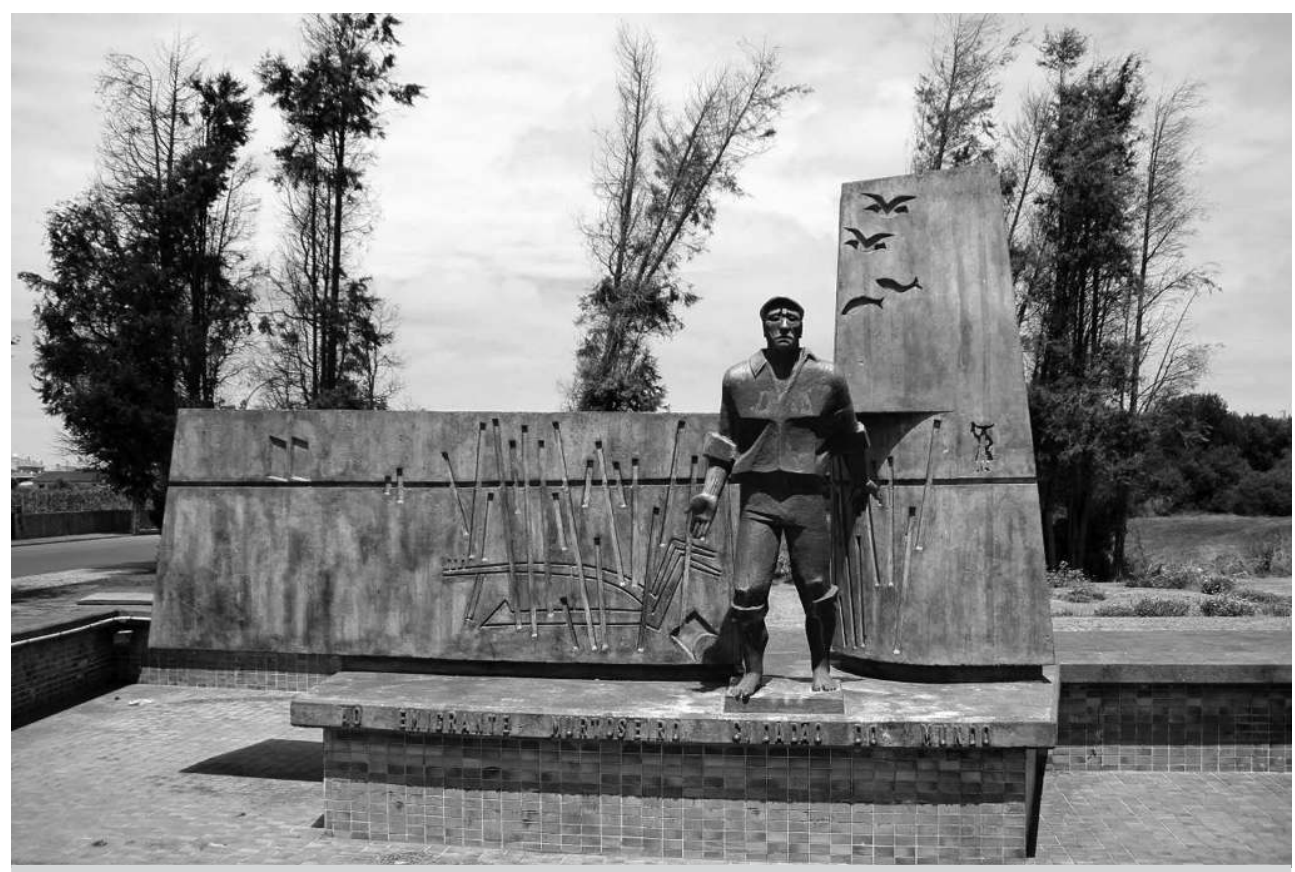

Figure 1 - Monument aux émigrants, Murtosa. Photo : G. Etienne, 2015.

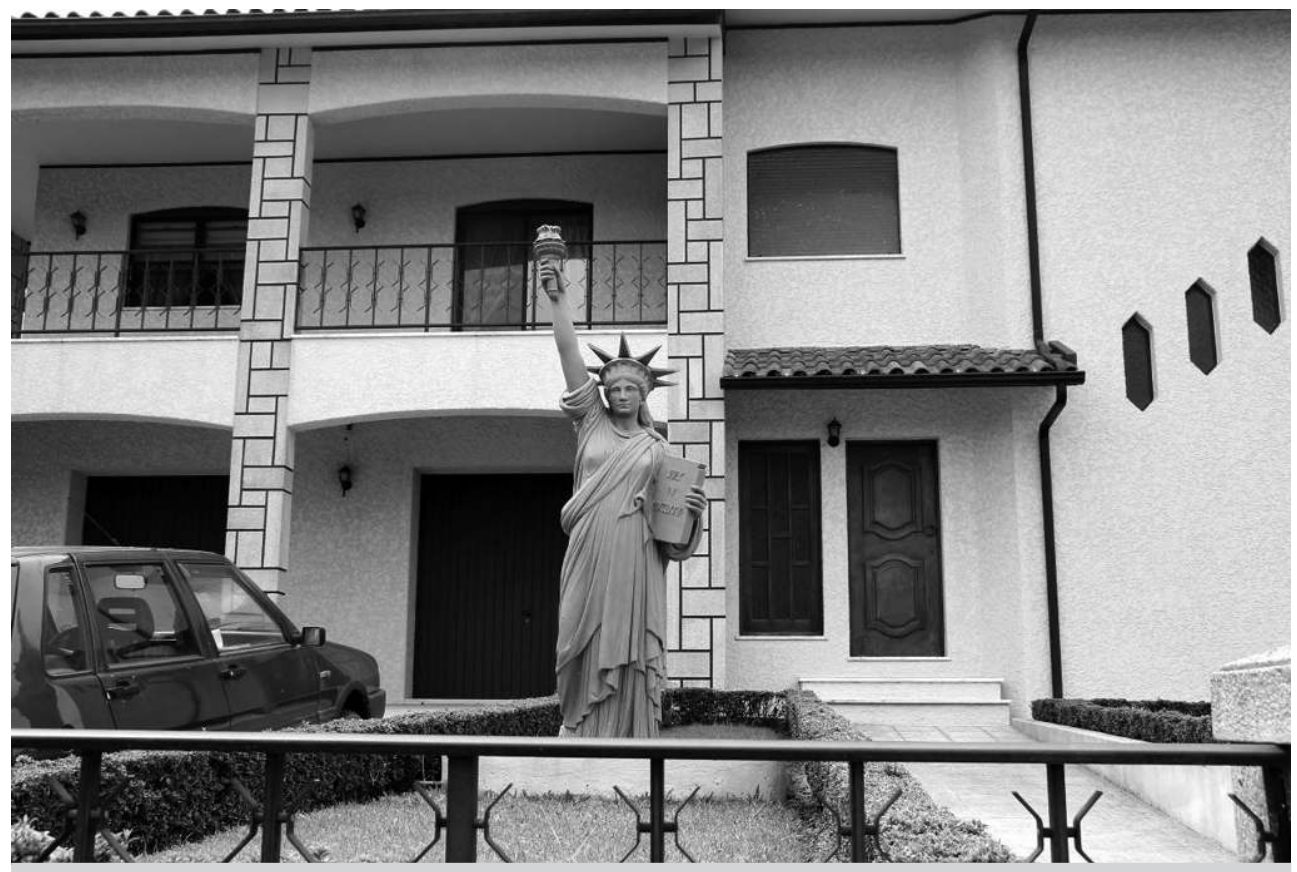

Figure 2 - Statue de la liberté dans un jardin, Murtosa. Photo : G. Etienne, 2015. 
nouvelles destinations apparaissent, comme la France et l'Allemagne. Toutefois, même lorsque le premier pays d'arrivée n'est pas les Etats-Unis, les murtoseiros y partent souvent dans un deuxième temps, surtout à partir de 1965 lorsque la politique américaine en matière d'immigration s'assouplit (Arroteia 1984a; Holton 2004).

La population du concelho de Murtosa chute ainsi de 21,9\% entre 1960 et 1970 (passant de 14.288 habitants à 11.160). Les mouvements de population sont toutefois inégaux selon les freguesias. Celle de Murtosa est particulièrement touchée, puisque dans cette même période sa population chute de $53,4 \% .{ }^{9}$ Les variations de population sont moins importantes pour les autres freguesias (Bunheiro perd 11,2\% d'habitants), voire positives (Monte gagne 19,6\%, et Torreira 0,13\%) (Arroteia 1984a : 71). Dans les pays d'arrivée, les émigrants deviennent majoritairement employés dans l'industrie et les services personnels (Arroteia 1984b).

Arroteia relève déjà en 1984 les liens entre les Etats-Unis et Murtosa et souligne le maintien des traditions et des fêtes lors desquelles on mange de l'anguille, spécialité et vecteur d'identité de Murtosa (1984a : 145). La pêche et la commercialisation de l'anguille sont en effet des activités typiques de la ville, et l'usine de conserverie de Murtosa (Comur) devient, dès sa création en 1942, caractéristique de la ville. ${ }^{10}$ Les échanges entre la localité d'arrivée et Murtosa permettent une meilleure compréhension locale de l'émigration et encouragent le respect pour les émigrants. Les remises des émigrants fourniront la principale source de richesse de Murtosa et contribueront pleinement au développement local (restauration de l'église, équipement de l'hôpital (Arroteia 1984a : 145-146), ce que reconnaît de nos jours la municipalité. ${ }^{11}$ Cela est sans compter les remises sociales (social remittances) théorisées par Peggy Levitt (1998), circulations d'ordre culturel, idéologique, pratique, aussi bien individuelles que collectives. Bien entendu, ces remises se transmettent différemment selon le réseau qu'elles mobilisent, la position sociale du transmetteur et de celui ou celle qui les reçoit et selon les modalités de la transmission. A Murtosa, la municipalité entretient ce passé migratoire ; beaucoup d'activités de la ville sont associées à l'émigration en tant que thématique centrale ou secondaire. L'exemple des liens entre Murtosa et La Riche illustre la façon dont la mise en lumière de liens individuels et collectifs entre les deux villes est désormais aussi intégrée à la mémoire collective.

9 Passant de 5779 à 2695 habitants (Arroteia 1984a).

10 Le site Internet du musée de la conserverie précise que l'anguille est la marque identitaire et marque de fabrique de Murtosa (<https://www.comur.com $>$, dernière consultation en décembre de 2017).

11 "Não será, pois, descabido, apontar a Emigração como um dos factores que mais ajudou a construir a matriz cultural e social da Murtosa e dos seus habitantes", < http://www.cm-murtosa.pt/Templat es/GenericDetails.aspx?id_object=5360 >, dernière consultation en décembre de 2017). 


\section{Que sont devenus nos migrants portugais?}

La Riche, petite ville située près de Tours, en France, entretient des contacts réguliers avec Estarreja (à quelques kilomètres de Murtosa, au Portugal) dans le cadre de leur jumelage depuis 1989. Divers événements sont organisés : échanges de délégations, échanges scolaires, fêtes communes. Du côté portugais, le jumelage d'Estarreja ne se limite pas aux habitants de la ville, mais capte également quelques habitants de la ville voisine, Murtosa. Comme nous l'avons vu, l'histoire de Murtosa est marquée par l'émigration vers les EtatsUnis, le Venezuela ou la France, mais pas particulièrement à destination de La Riche. Les liens de Murtosa se tournent plutôt vers la ville de Newark connue par le nombre de portugais, et notamment de murtoseiros, venus s'y installer (Holton 2005). Quant aux portugais venus en Touraine, et à La Riche en particulier, ils sont plutôt originaires de Nisa, dans l'Alentejo, ou du nord-est, mais peu de Murtosa ou d'Estarreja.

En 2014, le comité de jumelage et la municipalité de La Riche entament une recherche sur les portugais de la ville à laquelle j'ai participé. ${ }^{12}$ L'enquête a porté sur les traces de la migration portugaise à La Riche : que sont devenus les migrants, hommes et femmes, venus travailler dans les années 1960-70?

L'enquête de terrain fut réalisée sur la base d'entretiens avec les portugais ayant habité à La Riche, y habitant encore, mais aussi avec ceux qui les ont côtoyés, comme les employeurs ou des voisins. A l'issue de ce travail de six mois, une exposition a été présentée à la médiathèque de la ville début 2015 , en présence de portugais de la région ou venus spécialement du Portugal pour l'occasion (Wadbled, Etienne et Bertheleu 2015).

Durant l'enquête, l'une des membres du comité de jumelage prend connaissance de documentaires brossant des portraits d'émigrants réalisés par la mairie de Murtosa. L'un d'eux évoque la trajectoire de Rosa, une femme de 70 ans originaire de Murtosa, venue s'installer à Tours en 1969, avant de repartir au Portugal quelques années plus tard. Son parcours l'emmènera ensuite à Newark, aux Etats-Unis, durant près de 20 ans, pour enfin retourner à Murtosa, l'âge de la retraite approchant. Agostinho, habitant de Murtosa et membre de la délégation d'Estarreja, connaît justement Rosa et l'invite à venir témoigner lors du vernissage de l'exposition. Sachant que je m'intéraissais par les migrations portugaises, Agostinho évoque la fête des émigrants de Murtosa, organisée chaque année. Souhaitant poursuivre la recherche entamée en questionnant les parcours, les liens entre les personnes et entre les municipalités, je décide de participer à cette semaine de l'émigrant en août 2015. Agostinho profite

12 Cette enquête est menée conjointement par l'association Mémoires Plurielles et l'Université de Tours. Mémoires Plurielles est une structure régionale dont l'objectif est une connaissance approfondie des migrations dans la région Centre. L'enquête a été menée avec deux collègues, Hélène Bertheleu (Université de Tours) et Poleth Wadbled (Mémoires Plurielles). 
alors de ma venue pour intégrer au programme de la fête de l'émigration une présentation restituant le travail mené à La Riche.

Entre 2014 et 2018, l'enquête m'amène à participer aux fêtes de l'émigrant de Murtosa, mais aussi à d'autres événements, comme la fête patronale de S. Paio, en septembre. Dans une perspective ethnologique, des entretiens sont réalisés, dans l'agglomération de Murtosa et en France, avec des habitants de Murtosa qui ont migré vers différents pays, qui sont revenus à Murtosa ou qui, établis à l'étranger, reviennent de façon saisonnière. La migration ne concernant pas uniquement ceux qui partent, des entretiens menés avec celles et ceux qui n'ont jamais migré permettent d'éclairer les deux facettes d'un même phénomène : les représentations locales et les effets de la migration des uns sur la vie des autres. Enfin, la rencontre avec des acteurs publics (équipe municipale, prêtre, notables locaux) illustre la façon dont les trois thèmes de l'enquête - valorisation de la migration, développement municipal, réseau transnational sont articulés par celles et ceux qui, à un degré ou à un autre, ont une capacité d'expression et une visibilité sur la scène publique.

Commune d'un peu plus de 10.000 habitants en 2012, La Riche ne comptait que 6000 habitants dans les années 1960, au début de l'émigration importante vers la France. C'est l'une des villes où les portugais s'installent dans la région tourangelle, mais pas forcément dès leur arrivée en France. Leur installation s'y est faite au fur et à mesure pour plusieurs raisons. Les activités de maraîchage, importantes jusqu'aux années 1980, dans lesquelles les portugais sont embauchés, ainsi que les nombreux jardins familiaux permettant de cultiver fruits, légumes et mémoire du pays (Cardoso 2014) constituent des éléments attractifs. Le travail se trouve facilement dans cette période de plein emploi dans les grands chantiers du bâtiment. Certains, trop jeunes pour y être employés, travaillent d'abord dans le maraîchage, à La Riche notamment.

Les opportunités de travail ou de logement circulent de bouche-à-oreille (Charbit, Hily et Poinard 1997). La présence d'autres portugais de sa connaissance est souvent connue avant le départ et le voyage s'organise ainsi entre ici et là-bas. Les villes de départ et d'arrivée ont parfois rendu officielles ces relations par la reconnaissance publique, captant ainsi l'attention de "leurs" émigrants-immigrants : jumelages, invitations réciproques des édiles, monuments aux émigrants, etc.

Il n'existe pas à proprement parler de "quartier portugais " à La Riche et les familles d'origine portugaise sont de nos jours éparses dans la ville. Le stéréotype du portugais invisible semblerait, de premier abord, faire son chemin (Cordeiro 1999). ${ }^{13}$ Des indices, comme les azulejos sur les façades de maison, laissent, certes, parfois deviner que telle maison est habitée par des portugais. Quelques restaurants, épiceries ou bars soulignent également la référence 
portugaise par leur appellation ou leur décoration. Enfin, le jumelage avec Estarreja depuis 1989 officialise la relation et se concrétise par des échanges réguliers. Toutefois, la vie associative est concentrée sur d'autres villes de la région tourangelle : les groupes folkloriques, les clubs de football, les cours de langue, les célébrations annuelles du 25 Avril ou du jour des communautés sont essentiellement le fait d'associations basées dans des communes alentour, ce qui explique la faible dynamique associative à La Riche même, mis à part le Comité de Jumelage.

Les portugais habitant La Riche aujourd'hui n'y ont pas forcément passé leur vie : les trajectoires montrent des itinéraires faits de multiples déménagements en fonction des opportunités de travail. Les travailleurs s'installent d'abord dans des logements souvent vétustes du vieux Tours ou de la périphérie, parfois à la campagne et dans des baraquements précaires, comme à Larçay ou Veretz. Beaucoup de portugais originaires de Nisa s'installeront également à Azay-le-Rideau où se trouve un site industriel de transformation de bois qui embauchera nombre d'entre eux. L'étude de Jean Debon (1974a, 1974b) menée au début des années 1970 évoque la présence d'un pionnier originaire de Nisa qui aurait fait fortune, une trentaine d'années auparavant, dans la région ; histoire qui aurait accéléré le phénomène migratoire entre Nisa et la Touraine. ${ }^{14}$ En l'absence de statistiques pour les petites communes, il n'est pas possible de rendre compte avec précision du nombre de portugais venus s'installer à La Riche; un peu plus de 300 dans les années 1970, selon Debon. Beaucoup des portugais venus à La Riche viennent effectivement de Nisa, dans l'Alentejo, d'autres du nord (Póvoa de Lanhoso, Maia, Lamego) ou du centre (Leiria, Canedo, Sangalhos), mais très peu sont originaires de Murtosa ou d'Estarreja.

\section{Des relations transnationales asymétriques}

Quelles sont les raisons amenant deux villes portugaises, Estarreja et Murtosa, et une ville française, La Riche, à s'interroger sur leurs relations migratoires communes alors que, de toute évidence, les principales chaînes migratoires se sont entretenues avec d'autres localités? Du côté de La Riche, l'hypothèse d'un développement touristique et économique par l'entretien de ces liens ne peut être retenue : aucune structure locale ne révèle une telle volonté. Les quelques spectacles organisés à l'occasion des échanges de délégations n'autorisent pas une telle conclusion. Si le global et le local s'influencent mutuellement (Feldman-Bianco 2009), cela ne signifie pas que les relations transnationales revêtent la même importance ni les mêmes modalités d'expression pour les villes concernées.

14 Nous retrouvons également Nisa dans les portraits de plusieurs personnes présentés dans l'ouvrage de Robert Collet (2004). 
En effet, des asymétries peuvent exister entre deux localités nourries de liens transnationaux par des acteurs différents, aux pratiques et aux capacités différentes. A ce titre, certains concepts des études transnationales, comme celui de nationalisme de longue distance (long distance nationalism) (Anderson 1992), que Fouron et Glick Schiller définissent comme "a process of movement and settlement accross international borders in which individuals maintain or build multiple networks of connection to their country of origin while at the same time settling in a new country" (2002 : 171), sont particulièrement pertinents pour interroger ces relations, souvent plus fortes que le désir de retour au pays (Glick Schiller, Darieva et Gruner-Domic 2011 : 407). De ce fait, rien n'interdit de supposer que ces transnationalismes soient nourris de volontés diverses, accompagnant dans un cas le développement touristique (mais non exclusivement) et l'entretien d'une mémoire du pays dans l'autre, comme c'est le cas à La Riche. Cette relation entre Murtosa et La Riche est intégrée dans un maillage mémoriel, identitaire et touristique dont rend compte en partie la fête de l'émigrant de Murtosa.

\section{CONSENSUS SUR L'EMIGRATION : LA FETE DE L'EMIGRANT}

Les fêtes villageoises sont des moments importants pour les émigrés et leurs enfants qui, lors des retours saisonniers, peuvent réaffirmer l'attachement à leur village et maintenir un réseau de sociabilité (Hily 1996). Ces fêtes sont désormais, selon l'hypothèse développée par João Leal à propos des fêtes azoréennes, davantage axées sur les réseaux transnationaux que sur l'unité territoriale de la freguesia, en ce sens que leur calendrier se calque désormais sur celui du retour saisonnier des émigrants (Leal 2014a). L'anthropologue relève que les fêtes du Saint-Esprit sont aussi reconfigurées par l'émigration californienne, notamment avec l'introduction des reines dans les célébrations (Leal 2017). Cette perspective insiste sur la circulation des remises sociales, d'éléments idéels et culturels, entre ici et là-bas, qui, loin de remettre en cause l' “ authenticité des fêtes " comme certains le craignent, renforcent plutôt l'appartenance portugaise par l'intégration des portugais d'ailleurs.

Les fêtes des émigrants ont lieu dans tout le Portugal, généralement en été, de façon que les émigrants puissent justement y participer. Bien qu'elles ne fassent pas l'objet d'une organisation nationale, leur nombre et leur importance en font des événements des calendriers municipaux. ${ }^{15}$ La fête de l'émigrant de Murtosa est organisée tous les ans à la fin du mois de juillet / début août et durant plusieurs jours diverses activités rendent hommage aux émigrants à

15 Maria Beatriz Rocha-Trindade rapporte dans un entretien (Pignatelli et Rocha-Trindade 2014) la subvention du secrétariat d'Etat pour la réalisation de la série de films Festas, indice de l'importance politique de ces fêtes pour le gouvernement portugais. 
travers des événements aux dimensions festive, politique ou religieuse. La valorisation de l'émigration murtoseira y est abordée sous deux angles principaux : d'une part la célébration du phénomène migratoire et l'hommage rendu aux murtoseiros concernés, d'autre part la promotion de la région dans une perspective touristique et de développement à travers des "émissaires " multisitués. ${ }^{16}$ Les événements de la fête font varier la focale entre la freguesia de Murtosa, le concelho et la région de la Ria de Aveiro, en présentant des spécificités à chaque échelle géographique.

La municipalité " organise " le respect des émigrants et la valorisation de l'émigration, montrant le rayonnement de la culture locale, les réalisations des migrants, le maintien des liens et les échanges avec la communauté locale. L'émigration est rapportée à toutes les activités de la fête, mais ces animations sont aussi l'occasion de valoriser les particularités locales et régionales. Les formes que prennent les animations sont relativement similaires chaque année : concerts, expositions, projections de films, présentations de livres, messe des émigrants, marchés, régate. Les quatre premiers jours, en semaine, les animations ne sont proposées que le soir. Le week-end, elles commencent l'après-midi et se poursuivent jusqu'au soir : stands de nourriture, d'artisanat local, concerts sur les quais. Les thématiques abordées se rattachent à l'émigration, soit de manière directe - portraits d'émigrants, périodes et motifs des départs, lieux d'arrivées - soit de manière indirecte - vie locale, métiers et activités - et font varier l'échelle d'observation. En effet, ce sont tantôt des personnes particulières, des personnalités locales ou des émigrants qui sont au centre de la présentation, tantôt des faits plus généraux, comme l'émigration vers tel pays ou région.

\section{L'émigration abordée sans détour}

L'émigration est donc abordée de façon directe dans un certain nombre d'activités. Les livres tout comme les documentaires présentés évoquent la migration de leur auteur ou une étude sur l'émigration vers tels pays. Certains films sont produits par la municipalité, qui a en effet entrepris de réaliser des documentaires retraçant les parcours d'émigrants dans divers pays du monde.

En 2010, par exemple, a été présenté un livre de poésies sur l'émigration aux Etats-Unis par Augusto Amador et Fernando Silva (Amador et Silva 2010). Les deux auteurs, originaires de Murtosa, vivent actuellement aux Etats-Unis. Amador réside à Newark, où il fût dirigeant d'une grande entreprise de distribution énergétique et où il est aussi conseiller municipal depuis

16 Irène Dos Santos souligne l'importance de ces figures: "Cette politique diasporique s'appuie sur des acteurs sociaux particuliers, censés incarner des figures positives de la diaspora : l' 'émigrant à succès ' et surtout, dans le contexte intra-européen de plus lente mobilité socioprofessionnelle, les lusodescendants 'visibles ' dans les sociétés de résidence et potentiels ' ambassadeurs ' du Portugal à l'étranger" (2015: 146). 
1998, réussite professionnelle et politique qui constitue une certaine fierté locale, de la part des habitants comme du maire de Murtosa. ${ }^{17}$ Il revient en 2016 où il présente son nouveau livre de poésie, écrit en portugais et traduit en anglais, qui évoque avec nostalgie sa ville natale, mais aussi la vie à Newark. Amador reviendra en 2017 présenter un autre livre, co-écrit avec Carlos Faustino, murtoseiro lui-aussi.

Qu'il s'agisse d'expositions, de documentaires ou de livres, ces portraits mettent en lumière des personnes habitant à Murtosa ou originaires de la ville, afin de montrer leur réussite, mais aussi les spécificités d'une expérience migratoire, prestigieuse ou banale.

\section{Valoriser la localité, l'émigration en arrière-plan}

L'émigration est aussi traitée de façon indirecte, et c'est alors l'occasion de valoriser des savoir-faire, des artisanats locaux, souvent liées à la pêche et l'agriculture.

Les moliceiros, bateaux traditionnels à fond plat caractéristiques de la région de Murtosa, y sont à l'honneur. Ce sont d'une part des éléments identitaires forts, par ailleurs mis en exergue durant la période salazariste au regard de " l'art populaire " qu'ils représentaient. D'autre part, ils sont désormais une attraction touristique et patrimoniale de la ville (Sarmento 2009, 2010) saisie par les politiques locales. Ainsi ces bateaux trouvent place dans toutes les fêtes : les lancements d'ouvrages sur ce thème en 2012 et 2013 en témoignent, de même que les expositions de constructeurs et les régates qui clôturent toutes les fêtes de l'émigrant. Lors de ces courses, les pavillons portugais s'affichent à côté des pavillons américain, canadien, vénézuélien ou français, selon les pays où ont émigré les propriétaires. Les constructeurs reconnus de moliceiros font d'ailleurs souvent partie de ceux partis à l'étranger. Lors de la fête de 2015, l'exposition Coração na Ria, présentant le travail de deux constructeurs, soulignait le parcours luxembourgeois de l'un d'entre eux. Après avoir passé sa vie professionnelle au Luxembourg, cet homme, originaire de Pardilhó, à quelques kilomètres de Murtosa, est venu à Murtosa pour sa retraite. Il conserve des liens réguliers avec le Luxembourg, ses enfants ayant repris son entreprise sur place. C'est un savoir-faire local qui est ici valorisé à travers le parcours d'un constructeur, bien qu'au Luxembourg celui-ci ait exercé une toute autre activité, dans le bâtiment. Une chaîne de télévision régionale diffusée sur Internet lui consacre d'ailleurs plusieurs reportages en 2012, le suivant dans son entreprise luxembourgeoise et soulignant sa réussite.

17 Amador se réfère souvent à sa ville d'origine avec fierté lors de ses discours publics (Kimberly DaCosta Holton, communication personnelle). 


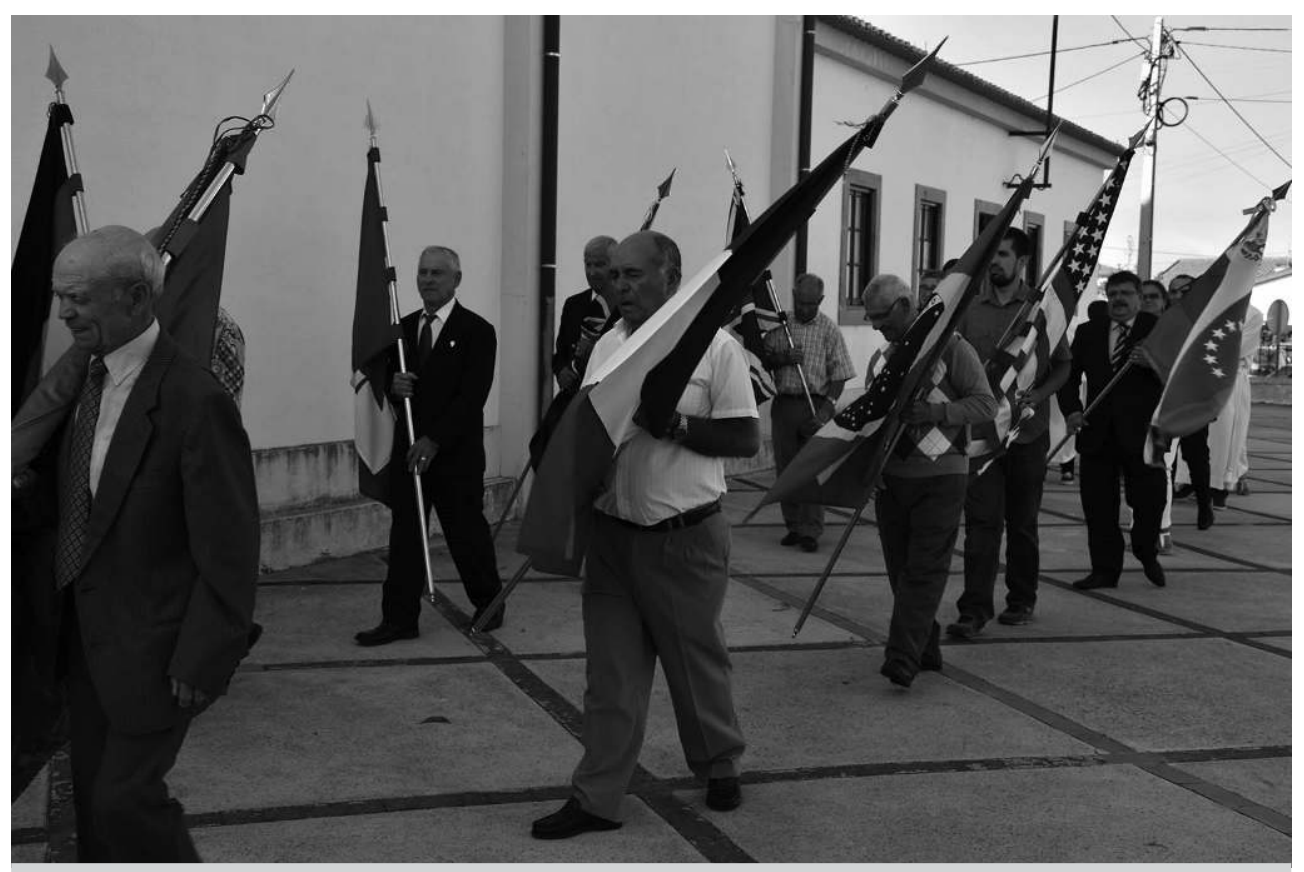

Figure 3 - Entrée des drapeaux, messe de l'émigrant. Photo : M. Esteves, município de Murtosa, 2016.

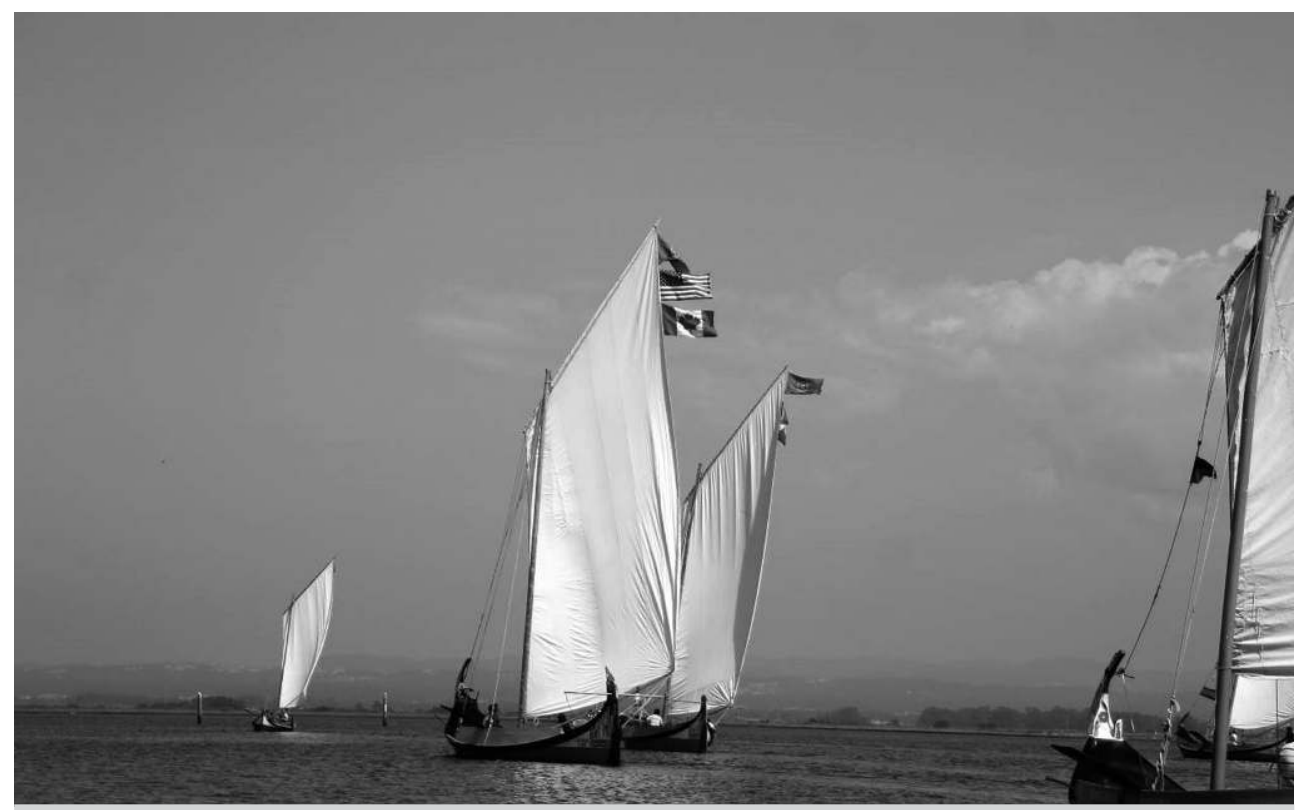

Figure 4 - Régate de moliceiros, drapeaux des pays d'émigration sur la voile, Murtosa. Photo : G. Etienne, 2016. 


\section{Messe et fête populaire pour terminer}

Le temps du week-end, la fête prend plus d'ampleur. Les festivités du samedi débutent en fin d'après-midi avec une messe dite de l'émigrant à l'église paroissiale. L'église est pleine, environ 300 personnes sont présentes, beaucoup restent debout faute de place. La moyenne d'âge est plutôt élevée et les jeunes gens sont minoritaires. L'entrée des drapeaux canadien, américain, allemand, vénézuélien et français se fait en grande solennité. Certaines années, les messes furent parfois célébrées par des prêtres invités de Newark (en 2005 et 2007) ou d'Elizabeth (en 2008). Puis les festivités se poursuivent sur les quais (Cais do Bico) et la soirée se termine par le concert d'artistes de variété portugais.

Enfin, les festivités du dernier jour débutent le matin, sur les quais également, avec un marché traditionnel. Beaucoup de monde vient y déjeuner, attendant le concert et la régate. Ainsi se clôt la semaine des émigrants. La " course cycliste des émigrants ", qui part du monument aux émigrants et réunit chaque année une centaine de cyclistes, a également lieu dans le cadre de cette fête.

A chacun des événements de la fête, les représentants de la mairie, en premier lieu le maire, interviennent publiquement pour valoriser l'émigration et la nécessité de rendre hommage à ceux qui sont concernés. Ces pratiques de reconnaissance ne se trouvent pas isolées. A l'échelle nationale, une reconnaissance politique de l'émigration est à l'œuvre, orchestrée par le gouvernement portugais, et de nombreux projets transnationaux voient le jour. Nous connaissons par exemple les rencontres européennes ou mondiales de lusodescendants qui ne manquent pas de questionner les modalités d'appartenance au Portugal ou au mythique empire de la Lusitanie. ${ }^{18}$ La sphère politique n'est d'ailleurs pas réservée aux instances étatiques ou municipales, et les associations, en France comme ailleurs, s'emparent elles-aussi de la question mémorielle des migrants portugais dans cette voie (Dos Santos 2015). Enfin l'Eglise, avec la Pastorale des migrants en France et la Obra Católica Portuguesa de Migrações au Portugal, confirme, par des structures organisées depuis les années 1960, son intérêt pour les migrations humaines en général. Les différents hommages rendus aux émigrants lors de la fête font donc intervenir des instances différenciées en d'autres circonstances, avec chacune leurs préoccupations spécifiques quant à l'émigration. ${ }^{19}$

18 Voir le numéro 9 de la revue Recherches en anthropologie du Portugal, publié en 2003 ("Lusodescendance : représentations, pratiques, enjeux").

19 Marie-Antoinette Hily relève une tendance à la dissociation du sacré et du profane dans les fêtes villageoises, sans arriver toutefois à la suppression de l'un au profit de l'autre. L'objectif est alors de contenter tous les villageois et " d'abolir le temps de la fête les distances en entraînant les gens dans un ensemble de réjouissances communes" (Hily 1996 : 509). 


\section{Des voix divergentes}

Maria Beatriz Rocha-Trindade (2010) relève la portée significative des fêtes annuelles en tant que lieux d'interactions entre les résidents et les émigrés et l'insertion d'éléments de la culture du pays d'arrivée dans la fête des migrants : dans la nourriture, les danses, les chants, etc. De fait se met à jour une certaine forme de compétition entre ceux d'ici et ceux d'ailleurs (Gonçalves 1996 ; Rocha-Trindade 2010 ; Dos Santos 2014) : compétition économique dans l'apport pour l'organisation des fêtes, compétition d'ordre récréative lors des jeux d'équipe, voire matrimoniale pour les jeunes gens (Charbit, Hily et Poinard 1997).

Le retour des migrants (définitif ou saisonnier) se traduit, à Murtosa comme dans d'autres régions, dans le style de construction des habitations, mais aussi dans un mélange de respect et de moquerie de la part de ceux qui sont restés. Les maisons des émigrants se différencient en effet des constructions plus traditionnelles, par l'utilisation de nouveaux matériaux, de nouvelles architectures, des revêtements en azulejos, ce qui modifie le paysage urbain (Arroteia 1984a : 149-153 ; Villanova, Leite et Raposo 1994) et attire aussi les critiques de ceux qui sont restés.

En 2015, une soirée est réservée à l'émigration portugaise vers la France lors de la fête de l'émigrant : la présentation de l'étude menée à La Riche, puis une projection du film La cage dorée de Rúben Alves (2012). L'appréhension du phénomène migratoire ne concerne plus ici directement Murtosa mais le Portugal en général. Les échanges qui suivent la présentation s'orientent vers l'assimilation des portugais émigrés et de leurs enfants. Un homme, installé en Amérique du Nord, m'interpelle en me questionnant sur ce que João Leal a appelé la peur du " ' syndrome de la deuxième génération ', syndrome structuré autour de la crainte, assez partagée, que la deuxième génération, perçue comme étant à mi-chemin d'une irréversible ' assimilation ', puisse venir remettre en cause la pérennité de la communauté luso-américaine " (Leal 2003 : 171). Cet homme interroge "la dilution du portugais dans des cultures plus fortes ", regrettant que ses petits-enfants arborent certes un maillot du club de football Benfica, mais ne parlent pas portugais. Selon lui, la langue serait le vecteur le plus fort de l'appartenance portugaise, en train de disparaître malgré les artifices connexes d'identification tels que les vêtements. Une idée précise, normative, de ce qu'est la “ portugalité " vient se confronter à la volonté d'intégrer les " communautés" de la diaspora. Cela montre les difficultés en matière d'identité et de transmission auxquelles font face certains émigrants et leurs descendants qui ne considèrent leurs liens avec le Portugal qu'à travers de certains éléments, comme la langue. Cela concerne particulièrement les secondes générations transnationales que Fouron et Glick Schiller définissent comme " all persons born into the generation after emigrants [that] have established transnational social fields who live within or 
are socialized by these fields, regardless of whether they were born or are currently living in the country of emigration or abroad " (2002 : 195). Si cette approche a le mérite de ne pas restreindre le transnationalisme au territoire pour l'élargir à la socialisation, il demeure que certains émigrants ne peuvent se résoudre à évincer du sentiment d'appartenance des éléments d'identification qu'ils perçoivent comme fondamentaux. Ce type de discours demeure toutefois peu légitime, du moins publiquement. Cet échange lors de la soirée provoque en effet le mécontentement de beaucoup de spectateurs qui y voient un débat sans fin, et plusieurs hommes de la salle interviennent pour faire cesser les questions de cet ordre.

Dans les discours et événements publics, l'émigration n'est donc pas critiquée et tout se passe comme si chacun avait bien intégré les raisons légitimes des départs. Les critiques sur l'émigration portent davantage sur des trajectoires individuelles ou bien sur les habitudes prises par certains groupes, raillés et caricaturés (par exemple " les américains ", “ les français ”, c'est-à-dire les portugais partis aux Etats-Unis, en France, etc.).

A Murtosa, la présence des migrants, des drapeaux, des langues qui se côtoient au marché ou au café, donne aussi l'occasion de moqueries et révèle des tensions qui sont masquées dans les temps forts de la fête. Les discussions révèlent les railleries, les noms donnés aux émigrants selon les pays où ils se sont installés en sont des indices. Ce sont aussi les propos sur les " avecs", nom donné aux portugais de France, ou encore les railleries sur le mélange d'anglais et de portugais, à propos de telle américaine demandant une "ice-cream gelado". Maria Beatriz Rocha-Trindade souligne justement ces discours oscillant entre plusieurs pôles: “Ainsi se manifeste une dialectique d'appartenance / différence, de proximité / distance, qui rend particulièrement intéressant l'environnement social des fêtes des migrants " (Rocha-Trindade 2010 : 51). Cette dialectique se relève dans les lieux de sociabilité comme les marchés ou les cafés. Un soir, Agostinho, qui parle presque couramment français, me prévient avant d'aller au café qu'il n'y parlera pas français. Il explique y avoir vu " des français " et que ceux-ci ont tendance à se moquer des portugais d'ici qui tentent de parler français. Ainsi le café est aussi un lieu de distinctions sociales et s'y rendre n'est pas une simple promenade (Rodrigues 2013 : 134 et sq.). Cela permet de voir et d'être vu, de se faire voir. Le marché tient un rôle similaire et lors d'une balade, mes informateurs disent être capables de reconnaître les émigrés et leurs pays d'arrivée. ${ }^{20}$ Les " françaises " mettraient des chaussures à talons, les " américains " seraient dans l'exaltation de leur richesse : "Les americains c'est show-off", me dit une habitante de Murtosa. Difficile de démêler ce qui relève de la connaissance réelle des familles émigrées par mes

20 Un informateur de Rodrigues (2013 : 151) relate la même capacité de reconnaissance des émigrés par leurs vêtements. 
interlocuteurs de déductions en fonction de leurs vêtements ou pratiques de plage. Ces déductions montrent toutefois une tendance à la naturalisation des distinctions sociales entre émigrés et non migrants (Rocha-Trindade 2006 ; Rodrigues $2013:$ 152). ${ }^{21}$

Les fêtes des émigrants sont donc à la fois des éléments d'inclusion des absents et des lieux de tensions entre émigrants et non migrants, notamment eu égard aux différentes significations qu'elles peuvent revêtir. ${ }^{22}$

\section{DE LA FETE DES EMIGRANTS A LA PROMOTION MUNICIPALE}

L'échelle oscille donc entre l'ici et l'ailleurs, la localité et la région, entre le respect et la moquerie, à tel point qu'il devient difficile de démêler et isoler la migration du reste des relations sociales. Pour la population locale, la migration fait partie intégrante de l'histoire locale et nationale. Certaines villes, comme Newark, entretiennent des liens privilégiés avec Murtosa mais, loin de s'en contenter, la municipalité cherche à explorer les autres endroits du monde où les murtoseiros, et les portugais en général, sont allés. Les événements de la fête de l'émigrant sont autant à destination des habitants de Murtosa que des portugais des autres régions du Portugal et du monde. Ainsi les informations sur la fête de l'émigrant sont relayées par les journaux portugais aux EtatsUnis, les sites Internet des " communautés" ou sur les pages personnelles. La municipalité de Murtosa en a bien conscience et utilise largement ces moyens de communication. La communication effectuée par la mairie se déploie en effet sur de nombreux sites Internet : mise en ligne des vidéos des fêtes de l'émigrant, de la fête patronale de S. Paio, du musée de la Comur, de portraits d'émigrants. Les réseaux sociaux de type Facebook sont investis aussi bien par le maire et ses adjoints que par le prêtre de Murtosa. Tous rapportent les événements locaux qui dépassent leur fonction politique ou religieuse. Ainsi le prêtre, sur sa page Facebook, publie aussi bien des textes ou des événements religieux que des vidéos de la fête de $\mathrm{S}$. Paio où on peut le voir danser en costume traditionnel lors du concours des groupes musicaux ambulants (rusgas). Autant d'outils permettent aux murtoseiros d'ailleurs de se tenir au courant des

21 Les moqueries sur les "avecs" sont une constante des blogs portugais durant les vacances d'été. Un site, parmi tant d'autres, titre "Les avecs sont arrivés " et en brosse un portrait satirique, passant en revue leur style vestimentaire, leurs pratiques à la plage, la musique qu'ils écoutent, leurs rapports à l'argent, les bijoux qu'ils portent, etc., distinguant les jeunes des plus âgés, les hommes des femmes. Cela entraîne une foule de commentaires appuyant ou rejetant ces moqueries, tant de la part de français que de portugais (<http://milcores.blogs.sapo.pt/8579.html >, dernière consultation en janvier de 2016).

22 Les interprétations de la fête peuvent également différer selon l'origine des migrants comme le relève, dans le contexte canadien lors du Portugal Day à Toronto, João Leal (2014b) à propos des continentaux et azoréens. 
événements de la ville, mais aussi aux portugais d'autres régions ou pays de prendre connaissance de la vitalité locale.

"C'est aussi une bonne promotion pour la commune. Parce que tous ceux qui regardent les vidéos qui sont partagées sur Facebook, par des gens d'ici, de Murtosa, c'est bon pour la commune, ça fait la promotion de la commune. Les émigrants, oui, ils viennent surtout pour les vacances, ils ne viennent pas exprès pour les festivités de São Paio, mais souvent ils restent quelques jours encore pour voir et participer. [...] Parce que souvent, quand on parle avec quelqu'un... Par exemple aux Etats-Unis : Ah, moi, je suis portugais. Alors tu habites l'Algarve? Non. Parce que le Portugal n'est pas seulement l'Algarve ! L'Algarve c'est la région la plus connue. L'Algarve, Lisbonne... Mais aujourd'hui les touristes cherchent des coins spécifiques, à cause de la promotion. Et si on fait la promotion du district d'Aveiro aux Etats-Unis, nous savons que beaucoup de ceux qui sont en retraite et qui cherchent à s'amuser pendant leurs vacances [penseront] pourquoi pas s'amuser ici ? S'ils viennent au Portugal en Algarve, pourquoi pas venir dans la région d'Aveiro ?" [Agostinho, 55 ans, habitant de Murtosa].

Les contacts maintenus dans les familles entre les murtoseiros d'ici et d'ailleurs sont l'occasion de promouvoir la ville, y compris auprès de ceux qui ne la connaissent pas vraiment. Rosa m'explique ainsi que, par l'intermédiaire des outils de communication comme Skype ou Facebook, elle peut parler avec ses petits-enfants aux Etats-Unis, même s'ils ne maitrisent pas le portugais : les traductions automatiques, bien qu'aléatoires, permettent ainsi de prendre des nouvelles régulièrement. Car la fête de l'émigrant n'est pas le seul événement à être rapporté à l'international. A l'étranger, les journaux relaient les principales informations locales, comme le journal Luso-Americano à Newark. Réciproquement, les journaux de Murtosa relatent les événements portugais à l'étranger ou des informations sur les personnes ayant émigré : l'anniversaire de mariage d'un couple parti à Newark, la première communion d'une fille ou fils d'un couple de murtoseiros aux Etats-Unis, le décès de tel autre parti au Brésil, l'obtention de diplômes par les petits-enfants, etc. Ces encarts permettent à chaque fois de rappeler l'origine des personnes, leurs parcours migratoires et leur réussite à l'étranger.

Au niveau politique, le tourisme occupe également une place importante dans les discours comme dans les œuvres entreprises. Le tourisme qui se développe tout au long du $\mathrm{XX}^{\mathrm{e}}$ siècle en vue d'attirer les étrangers va aussi cibler les portugais de l'étranger avec le développement du tourisme de la nostalgie (mercado de saudade, turismo de saudade). Cette prise en compte s'observe à l'échelle du Portugal en général et le tourisme est finalement envisagé dans ce contexte de transnationalisme et de retour des émigrants. Laéroport de Porto, au mois 
d'août, affiche ainsi aux arrivées un espace d'accueil particulièrement explicite, un grand panneau imitant le style des azulejos sur lequel figure l'inscription "Bem-vindo a Portugal. Festa do emigrante ". Sur le fond de ce panneau estampillé de l'entreprise Vinci figurent des motifs représentant les spécificités portugaises que les touristes s'attendent à trouver : le soleil, une bouteille, un château, une guitare, un tramway, un ballon... Les passagers sont par ailleurs invités à déguster des spécialités portugaises.

Du côté des pays d'émigration, le tourisme devient un support privilégié de la promotion culturelle. Par exemple, les " I ers Etats Généraux de la Lusodescendance ”, organisés par l'association Cap Magellan à Paris, en janvier 2017, comportaient trois axes de travail dont "la promotion culturelle via le tourisme et la mémoire ". ${ }^{23}$

Cette mise en tourisme de la migration de retour n'est d'ailleurs pas propre au Portugal et des auteurs, tel João Luís Jesus Fernandes (2007-2008), estiment que le tourisme européen dépend aussi de la particularité européenne en termes d'émigration et de diaspora. Ainsi, l'émigration des irlandais aux EtatsUnis (XIXe - début XXe siècle) a créé un tourisme de la nostalgie ou tourisme de la mémoire : les descendants d'irlandais vont, par exemple, visiter Ellis Island avec l'idée que leurs ascendants sont passés par là.

A Murtosa, le tourisme est décliné dans de nombreuses sphères (tourisme de l'environnement, rural et d'habitation, de la nature, cyclotourisme, tourisme culturel, nautique, de patrimoine, religieux...) $)^{24}$ et devient aussi un enjeu électoral. Par exemple, à l'occasion des élections locales de 2017, les candidats sont invités à s'exprimer sur le sujet, et notamment l'importance de maintenir l'attractivité touristique tout au long de l'année. Le candidat du Partido Socialista, Jorge Barcelar, met au premier plan l'identité unique que Murtosa tire de la Ria de Aveiro, notamment les moliceiros qu'il s'agit de promouvoir par davantage de construction et de visibilité. ${ }^{25}$ C'est également la gastronomie (l'anguille, la viande de race bovine Marinhoa), ${ }^{26}$ les fêtes thématiques et la

23 Voir le compte-rendu de ces états généraux sur le site de l'association (<http://capmagellan.com/e tats-generaux-de-lusodescendance $>$, dernière consultation en octobre de 2018).

24 Ces termes sont tous relevés dans le journal O Concelho da Murtosa.

25 "Temos que atrair os grandes agentes turísticos nacionais e internacionais criando infraestruturas de apoio que satisfaçam quem nos visita [...] Temos que saber atrair regularmente os agentes turísticos ao longo de todo o ano. E como o devemos fazer? Sem dúvida promovendo a nossa identidade, que nos diferencia, positivamente, de outras regiões e nos torna únicos no mundo. Assim devemos promover o barco moliceiro colocando-o durante todo o ano na ria; temos que incentivar a construção de mais barcos" ("Autárquicas 2017, entrevista com o candidato do Partido Socialista, Dr. Jorge Barcelar", O Concelho da Murtosa, 31 de agosto de 2017, pp. 4-5).

26 Par exemple la Feira Agrícola da Murtosa qui, depuis 2014, met à l'honneur l'agriculture et particulièrement l'élevage bovin dans une ambiance de fête, d'artisanat, de folklore. Là-encore, le rapport à l'agriculture dépasse la seule activité professionnelle, puisque le concours "tem a pretensão de exaltar a cultura associada à ruralidade, num Concelho onde o sector primário - e a actividade agrícola [continua] 
nature qu'il faut mettre en avant de la promotion touristique. Le candidat réélu du Partido Social-Democrata, Joaquim Baptista, affirme quant à lui que la Ria de Aveiro occupe une place centrale dans la vie politique locale, si bien qu'il “ est impossible de penser la pêche, l'agriculture, le tourisme, l'écologie et beaucoup d'autres choses sans penser à la Ria ". ${ }^{27}$

Cette identité unique que fournit la Ria de Aveiro, les moliceiros ou l'anguille de Murtosa s'accompagne d'une autre forme d'identité liée au Portugal en général mais reconstruite localement en fonction des attentes des touristes. Par exemple, le fado tend à se développer dans la ville, comme le relève cette femme, épouse d'un homme originaire de Bunheiro :

"Alors maintenant le fado ça devient une culture collective. Même ici, je suis étonnée, ils commencent à faire des concerts de fado. Tout arrive. Mais c'était vraiment pas la culture. C'était vraiment une autre musique. [...] C'est seulement depuis un an, peut-être deux, qu'ils font des trucs de fado. Maintenant ils voient bien que l'image du Portugal à l'extérieur, une des images positives, c'est le fado. Alors pourquoi on n'en aurait pas?"

Le maire adjoint précise explicitement en entretien que les fêtes de l'émigrant sont importantes au regard de la promotion de la ville qu'elles apportent, au Portugal comme à l'étranger. L'idée que les portugais de l'étranger puissent venir à Murtosa après avoir visité leur famille dans d'autres régions est exprimée en termes touristiques et donc économiques. Le migrant transnational serait-il un touriste incognito ? La politique municipale en matière de tourisme illustre bien l'intérêt pour ce secteur avec les grandes rénovations urbaines, ${ }^{28}$ notamment dans la freguesia de Torreira, où se trouvent les plages. Les liens entre ces deux formes de mobilité que sont le tourisme et la migration semblent donc se complexifier (Dehoorne 2002) à travers le tourisme des retraités et des enfants, voire petits-enfants de migrants revenant au pays. D'ailleurs, les pratiques des plus jeunes semblent davantage se rapprocher du tourisme que de la sociabilité familiale privilégiée par les plus âgés (Cavaco 2009). Mais le développement touristique de la ville passe aussi par d'autres canaux que la seule émigration,

[continuação] em particular - assume grande importância económica e social, contribuindo desta forma para a afirmação da Murtosa neste domínio, no contexto regional" (< http://www.cm-murtosa. pt/Templates/GenericDetails. aspx?id_object=7212 >, dernière consultation en juin de 2018).

27 "A nossa ria se assume como um elemento central no processo de decisão no nosso concelho pois é impossível pensar em pesca, agricultura, turismo, ambiente e muitas outras áreas sem pensar na Ria” ("Autárquicas 2017, entrevista com o candidato do Partido Social Democrata, Eng. Joaquim Baptista", O Concelho da Murtosa, 31 de agosto de 2017, p. 6).

28 Le nouveau camping de Torreira, construit en $201 \mathrm{l}$, accueille des campeurs sur plus de $32.000 \mathrm{~m}^{2}$. Des habitants de Murtosa et alentours s'y rendent également, notamment lors des fêtes de S. Paio, pour être au plus proche des festivités et bénéficier d'un autre cadre que celui du quotidien. 
des plus classiques, comme l'attrait des plages, aux plus originaux, comme le cyclotourisme. La municipalité insiste sur cette image de ville cycliste avec la création de circuits touristiques à vélo, des courses régulières, comme la course cycliste des émigrants, ou des événements tels les rencontres européennes de cyclotourisme en 2014, réunissant des cyclistes de toute l'Europe.

\section{CONCLUSION}

Partant d'une enquête menée dans la région de Tours, en France, j’ai souhaité montrer les relations entre villes et habitants qui tendent à se créer au-delà des chaînes migratoires reliant les territoires. J'ai ainsi tracé la généalogie des liens entre deux villes, La Riche et Estarreja, qui finalement embrassent d'autres communes, comme Murtosa, par l'intermédiaire des membres du comité de jumelage. L'étude menée à La Riche questionnait les trajectoires des migrants portugais et relevait les liens maintenus entre ici et là-bas.

J'ai insisté sur la diversité des sphères de la vie sociale mobilisées sur fonds de passé migratoire lors de la fête de l'émigrant de Murtosa, telles que le politique, le religieux, le loisir, l'art ou l'ethnographie. Ce sont de fait autant d'interprétations qui semblent se dessiner autour du fait migratoire, allant du consensus au dissensus. Parmi les effets escomptés, celui de la promotion municipale n'est pas négligeable. Au-delà des seuls émigrants, ce sont finalement tous les portugais de l'étranger que la municipalité de Murtosa cherche à capter, parfois en modulant les réalités migratoires. La présentation que j'ai faite à Murtosa sur les portugais installés à La Riche, s'est ainsi vue traduite, dans le journal local, en une démonstration de la présence de murtoseiros dans la région tourangelle, où de nombreux murtoseiros ont travaillé et travaillent encore. ${ }^{29}$ Dans le même article de journal, Agostinho s'est vu dénommé le "ministre des affaires étrangères" de Murtosa, en raison de ses liens avec la France et de mon accompagnement lors de la fête. Enfin, je fus moi-même envisagé comme un intermédiaire potentiel entre Murtosa et les portugais de la région tourangelle, le maire étant intéressé par la promotion de la ville que je pourrais faire par ma recherche. Je suis d'ailleurs reparti de Murtosa avec des informations touristiques à destination des larichois et tourangeaux, de même que des effets plus personnels pour des connaissances établies avec des larichois. Le transnationalisme passe donc aussi par les chercheurs qui l'étudient. Il serait bien sûr exagéré de conclure que l'émigration n'est envisagée que d'un point de vue promotionnel par les municipalités. Les relations passent certes par d'autres canaux que la seule sphère politique. En revanche, il est notable de souligner que la captation en étoile des migrants de Murtosa ou d'ailleurs

29 Voir " O mérito da diáspora sempre celebrado e reconhecido: Murtosa celebrou o mais emotivo ciclo festivo do ano ", O Concelho da Murtosa, 31 août de 2015, p. 15. 
par les villes constitue aussi un enjeu de visibilité à une échelle européenne ou mondiale et, a fortiori, un vecteur de promotion municipale dans le secteur du tourisme.

\section{BIBLIOGRAPHIE}

AMADOR, Augusto, et Fernando SILVA, 2010, (Re)Cantos da Viagem. Newark, s.n.

ANDERSON, Benedict R., 1992, "Long-distance nationalism: world capitalism and the rise of identity politics", The Wertheim Lecture. Amsterdam, CASA.

ARroteIA, Jorge, 1984a, Os Ílhavos e os Murtoseiros na Emigração Portuguesa. Aveiro, Associação de Defesa do Património Natural e Cultural da Região de Aveiro.

ARROTEIA, Jorge, 1984b, "Ílhavo e Murtosa: dois casos da emigração portuguesa”, em Emigração e Retorno na Região Centro. Coimbra, Comissão de Coordenação da Região Centro, Ministério da Administração Interna, 123-148.

BRETTELL, Caroline, 2003, Anthropology and Migration: Essays on Transnationalism, Ethnicity, and Identity. Lanham, AltaMira.

BRETTELL, Caroline, 2008, "Adjustment of status, remittances, and return: some observations on 21 st century migration processes", City \& Society, 19 (1) : 47-59.

BRETTELL, Caroline, 2015 [2000], “Theorizing migration in anthropology: the cultural, social and phenomenological dimensions of movement", dans C. Brettell et James Hollifield (dirs.), Migration Theory, Talking across Disciplines. New York, Routledge, 148-197.

CARDOSO, Isabel Lopes, 2014, “'Quintal é rei em casa de português': jardins e hortas em contexto migratório", em H. Pires et al. (dirs.), Jardins, Jardineiros, Jardinagem. Braga, Centro de Estudos de Comunicação e Sociedade da Universidade do Minho, 39-77.

CAVACO, Carminda, 2009, "Turismo sénior: perfis e práticas”, COGITUR : Jornal of Tourism Studies, $3:$ 33-64.

CHARBIT, Yves, Marie-Antoinette HILY, et Michel POINARD, 1997, Le va-et-vient identitaire : Migrants portugais et village d'origine. Paris, PUF-INED.

COHEN, Jeffrey H., 2004, The Culture of Migration in Southern Mexico. Austin, University of Texas Press.

COLlET, Robert, 2004, Portugais en Touraine de 1930 à aujourd'hui. Saint-Cyr-sur-Loire, A. Sutton.

CORDEIRO, Albano, 1999, “Les Portugais, une population 'invisible' ? ”, dans Philippe Dewitte (dir.), Immigration et intégration : l'état des savoirs. Paris, La Découverte, 106-111. DEBON, Jean, 1974a, “Les portugais en Touraine ”, Norois, 82 : 195-208.

DEBON, Jean, 1974b, “Les portugais en Touraine ”, Norois, 83 : 379-392.

DEHOORNE, Olivier, 2002, “ Tourisme, travail, migration : interrelations et logiques mobilitaires ", Revue européenne des migrations internationales, 18 (1) : 7-36.

DOS SANTOS, Irène, 2014, "Confrontação com o país de origem e redefinição das identificações: descendentes de migrantes portugueses no contexto intraeuropeu (França-Portugal)", InterDisciplinary: Journal of Portuguese Diaspora Studies, 3 (2) : 433-459. 
DOS SANTOS, Irène, 2015, “ Identité collective et construction politique d'une diaspora : usages du passé dans la migration portugaise ”, dans M. Amar, H. Bertheleu et L. Teulières (dirs.), Mémoires des migrations : temps de l'histoire. Tours, Presses universitaires François Rabelais, 139-157.

FELDMAN-BIANCO, Bela, 2009, “'A taste of Portugal’: transmigração, políticas culturais e a mercantilização da 'saudade' em tempos neoliberais”, Ler História, 56 : 105-1 19.

FELDMAN-BIANCO, Bela, et David HUSE, 1995, "Entre a saudade da terra e a América: memória cultural, trajectórias de vida e (re)construções de identidade feminina na intersecção de culturas”, Ler História, 27-28 : 45-73.

FERNANDES, João Luís Jesus, 2007-2008, “Território, cultura e diversidade da oferta turística na Europa”, Cadernos de Geografia, 26-27 : 53-65.

FOURON, Georges E., et Nina GLICK SCHILLER, 2002, “The generation of identity: redefining the second generation within a transnational social field", dans P. Levitt et M. Waters (dirs.), The Changing Face of Home: The Transnational Lives of the Second Generation. New York, Russell Sage Foundation, 168-208.

GLICK SCHILLER, Nina, Tsypylma DARIEVA, et Sandra GRUNER-DOMIC, 201 1, "Defining cosmopolitan sociability in a transnational age: an introduction", Ethnic and Racial Studies, 34 (3) : 399-418.

GONÇALVES, Albertino, 1996, Imagens e Clivagens: Os Residentes Face aos Emigrantes. Porto, Afrontamento.

HILY, Marie-Antoinette, 1996, “ Immigrés et espace d'origine : le village des portugais ”, Espace, populations, sociétés, 14 (2-3) : 507-512.

HOLTON, Kimberly DaCosta, 2004, “Dancing along the in-between: folklore performance and transmigration in Portuguese Newark", Portuguese Studies Review, 11 (2) : 153-182.

HOLTON, Kimberly DaCosta, 2005, Performing Folklore: Ranchos Folclóricos from Lisbon to Newark. Bloomington, Indiana University Press.

KANDEL, William, et Douglass S. MASSEY, 2002, "The culture of Mexican migration: a theoretical and empirical analysis", Social Forces, 80 (3) : 981-1004.

LEAL, João, 2003, “Deuxième génération : visibilité et invisibilité ”, Recherches en anthropologie du Portugal, 9 : 167-174.

LEAL, João, 2014a, “Transnacionalidade, etnicidade, sincretismo: viagens atlânticas das Festas do Divino", Revista de Políticas Públicas, numéro spécial : 351-358.

LEAL, João, 2014b, "What's (not) in a parade? Nationhood, ethnicity and regionalism in a diasporic context”, Nations and Nationalism, 20 (2) : 200-217.

LEAL, João, 2017, "Ritual, transnationalism and social remittances: two way travels of the Holy Ghost”, Análise Social, LII (225) : 760-781.

LEVITT, Peggy, 1998, "Social remittances: migration driven local-level forms of cultural diffusion”, International Migration Review, 32 (4) : 926-948.

MONTEIRO, Miguel, et Maria Beatriz ROCHA-TRINDADE, 2007, "Emigração e retorno: imagens cruzadas num webmuseu e o papel da tecnociência no caso de www.museu-emi grantes.org", dans F. Sousa et I. Martins (dirs.), A Emigração Portuguesa para o Brasil. Porto, Afrontamento, 437-448.

PIGNATELLI, Marina, et Maria Beatriz ROCHA-TRINDADE, 2014, "Entre o idealizado e o exequível: memórias da primeira mulher antropóloga portuguesa”, Etnográfica, 18 (2) : 443-455. 
ROCHA-TRINDADE, Maria Beatriz, 2006, "Recriação de identidades em contextos de migração”, dans C.T. Lucena et N.M.M. Gusmão (dirs.), Discutindo Identidades. São Paulo, Humanitas, 75-90.

ROCHA-TRINDADE, Maria Beatriz, 2010, "Associativismo em contexto migratório", Revista Migrações, $6: 39-58$.

RODRIGUES, Weslei Estradiote, 2013, “Meu Portugal é a Aldeia”: Etnografia de Uma Dinâmica de Circulação Migratória e Práticas Transnacionais. São Paulo, Universidade de São Paulo, dissertation de maîtrise.

RSM - Rede Social da Murtosa, 2006, Diagnóstico Social do Concelho da Murtosa. Murtosa, Rede Social da Murtosa, Conselho Local de Acção Social.

SARMENTO, Clara, 2009, "Folk culture and political power: practices and representations of moliceiro culture in Portugal", Cultural Sociology, 3 (3) : 347-376.

SARMENTO, Clara, 2010, "All the world in a boat': Portuguese folklore, common sense and ideology”, Folklore, 121 (3) : 268-291.

TOMÉ, Alice, 2009, “Art et mémoire : éducation, émigration, lusophonie ”, Scientific Annals of the "Alexandru Ioan Cuza" University, Iaşi: Sociology and Social Work, n. s., 2 (1) : 183-196.

TOMÉ, Alice, Teresa CARreIrA, et Francisco CARrEIRA, 2000, Mitos, Arte, Educação: Monumentos ao Emigrante em Portugal. Lisboa, Edições 70.

VILLANOVA, Roselyne de, Carolina LEITE, et Isabel RAPOSO, 1994, Maisons de rêve au Portugal. Paris, Créaphis.

WADBLED, Poleth, Guillaume ETIENNE, et Hélène BERTHELEU, 2015, Familles portugaises à La Riche : Souvenirs de migration et formes d'ancrage / Famílias Portuguesas em La Riche: Memórias de Migração e Formas de Ancoragem. La Riche, Comité de jumelage, rapport d'étude.

\begin{tabular}{ll}
\hline Receção da versão original / Original version & $2017 / 08 / 23$ \\
Receção da versão revista / Revised version & $2018 / 10 / 06$ \\
Aceitação / Accepted & $2018 / 11 / 28$ \\
Pré-publicação online / Pre-published online & $2019 / 12 / 31$
\end{tabular}

